Original Research Paper

\title{
A Real-Time Optimization Approach to the Efficiency of a Photovoltaic System by a Hybrid Tracking Technique using LabVIEW
}

\author{
${ }^{1}$ Mohamed Redha Rezoug and ${ }^{2}$ Rachid Chenni \\ ${ }^{1}$ Departement of Electrical Engineering, Université Kasdi Merbah Ouargla, Algeria \\ ${ }^{2}$ MoDERNa Laboratory, Mentouri University-Constantine, Algeria
}

Article history

Received: 17-02-2018

Revised: 28-04-2018

Accepted: $12-05-2018$

Corresponding Author: Mohamed Redha Rezoug Departement of Electrical Engineering, Université Kasdi Merbah Ouargla, Algeria Email: redha77.rmr@gmail.com

\begin{abstract}
Among the lines of research on improving the efficiency of a photovoltaic panel, sun tracking techniques occupy a large part. In this article, an extension of Laboratory Virtual Instrument Engineering Workbench (LabVIEW); LabVIEW Interface For Arduino (LIFA) is used. It allows the driving of an Arduino board from LabVIEW, on which a collaboration technique between two categories of pursuit is developed: The first is sensory and the second is pre-programmed. This hybrid two-axis technique is characterized by impulse control strategist with pre-programmed control and dual sensory correction. The results reveal that the hybrid tracking system gave an additional energy gain of about $36 \%$ compared to a fixed photovoltaic panel system. Thus, it has practically a percentage increase of 2 and $2.3 \%$ respectively to the two techniques used.
\end{abstract}

Keywords: Hybrid Tracking, Pre-Programmed, Sensory Correction, Azimuth

\section{Introduction}

For a very long time, man had sought to use the energy emitted by the sun, the closest star to earth. Most uses are direct as in agriculture, through photosynthesis or in various drying and heating applications, both artisanal and industrial. This energy is available in abundance all over the terrestrial surface and in spite of a significant attenuation during the crossing of the atmosphere; an important quantity arrives at the surface of the ground. Thus, it is possible to rely on $1000 \mathrm{~W} / \mathrm{m}^{2}$ in temperate zones and up to $1400 \mathrm{~W} / \mathrm{m}^{2}$ when the atmosphere is slightly polluted with dust or water (Arslan et al., 2014; Ya'u, 2017).

The solar flux received at ground level thus depends on several parameters such as:

- The orientation, nature and inclination of the earth's surface

- The latitude of the collection site, its degree of pollution as well as its altitude

- The period of year

- The considered moment in the day

Photovoltaic panels have the quality of being a natural resource of clean and sustainable energy. Unfortunately their poor performance (less than 20\% under the best conditions) (Chong and Wong, 2009; Eke and Senturk, 2012) due to the influence of several factors (day alternation - night, wind, lighting, temperature ...) urges us to amplify the radiant energy reception capacity and thus lead the photovoltaic generator to a maximum operating point (V-I). For this, several correction systems are used. They focus their interest on the pursuit of the sun.

The movement of the earth around itself and around the sun poses the problem of the variation of solar illumination. Hence, implementing a mechanical tracking system has become a necessity.

Two main categories characterize solar trackers. The first is known under the name of active trackers and the second as passive trackers (Senpinar and Cebeci, 2012; Yougeshwarsingh and Vishwamitra, 2014). These must be equipped with tracking systems that provide orientation around one or two axes to maximize the solar luminous flux over the active surface of the panel (Ya'u, 2017; Zhang et al., 2013). The popular configurations used in solar tracking are bi-axial (elevation and azimuth) and tilt-roll (polar) Fig. 1, which produce additional energy gain over fixed-tilt devices (Eke and Senturk, 2012; Salsabila et al., 2013; Walsh et al., 2017). The accuracy of tracking using electrical or electronic devices makes active trackers appreciated. They are 
subdivided into two systems: The first in open loop is based on algorithms that use mathematical formulas (the equations of time) to predict the steps of sun movement, the second in closed loop uses sensors according to their functions in order to capture at all times the position of the sun and to correct the deviation angle of the luminous flux which makes it an energy consumer (Carlos et al., 2017; Fabienne et al., 2015; Saban et al., 2015).

For a better correction of the position of the photovoltaic panel, it is necessary to take into account the rotation of the earth around its axis. It describes an elliptical trajectory corresponding to the day-night cycle. The duration of sunshine during a day varies throughout the year and must be known to determine the limits of the daily correction which can be considered as optimal sunshine duration (sunrise and sunset).

The paper proposes a hybrid tracking algorithm implanted in an Arduino to execute collaboration between the two systems mentioned previously (the closed loop and the open loop). Through a LIFA extension, an advanced recognition of the position of the sun and the sunrise and sunset time is used by pre-programming the equations of time by simplifying the control and minimizing the consumption of the sensors during the orientation process by double correction via sensors under azimuth tracking. The experimental results led to a number of additional correction steps in the form of intervention of the orientation sub-programmes. These sub-programmes are produced by the errors of the first correction.

Using this two-axis hybrid orientation technique, the amount of direct solar radiation as well as the energetic capacity received by the photovoltaic panel has increased by $37.5 \%$, while the energy consumption of the two-axis tracker is reduced by about $36 \%$.

\section{Modeling the Trajectory by Time Equations}

The majority of solar applications require knowledge of the apparent movement of the sun for a given location on the Earth's surface, characterized by its latitude, longitude, and distance from the sun.

The correction of the error related to the change of the position and the distance of the sun with respect to the photovoltaic module making it possible to keep the latter always perpendicular to the solar irradiations, obliges the use of a correction system with two axes, the first concerns the variation day-night, the second concerns the variation of the seasons (the point of sunrise compared to the zenith) (Alexandru and Tatu, 2013; Radu and Mihai, 2014).

A main angle must be taken into account for the modelling of the trajectory. It is the declination which is the angle that the sun makes at the maximum of its course (solar noon) with respect to the equatorial plan. It varies between $-23.45^{\circ}$ on December 21 st and $+23.45^{\circ}$ on June 21st, (Senpinar and Cebeci, 2012). For this, it is important to establish an equation connecting the position of the sun relative to the perpendicular to the plan of the photovoltaic module. Figure 1 shows the angles involved to locate the plan of the solar module relative to the position of the sun (Arslan et al., 2014; Cheng et al., 2013).

The Table 1 gives the values of elevation and azimuth angles as well as sunrise and sunset for a day. The whole is calculated by the hybrid algorithm via the Arduino board in the form of a database depending on the location.

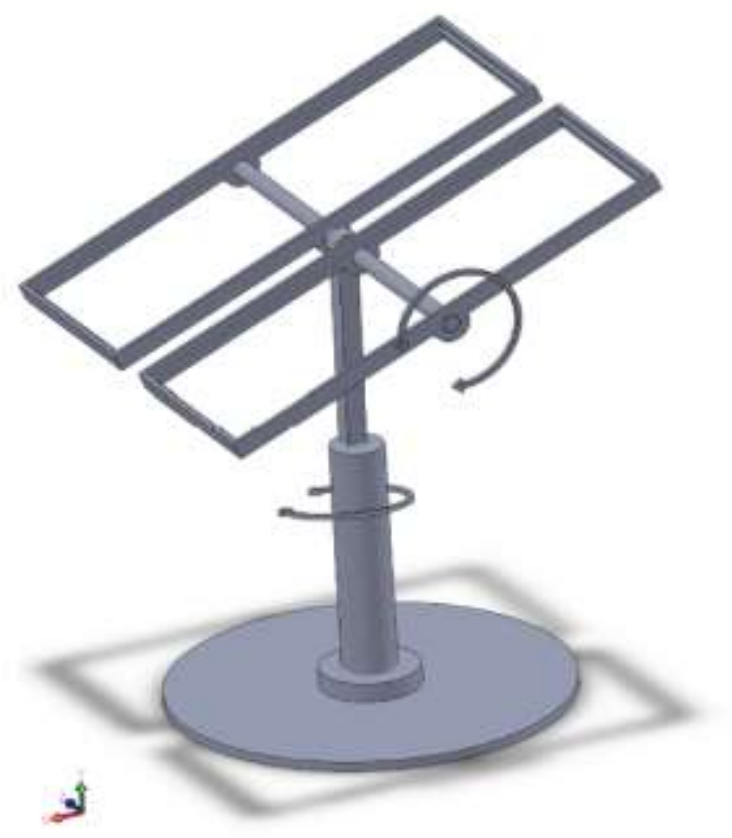

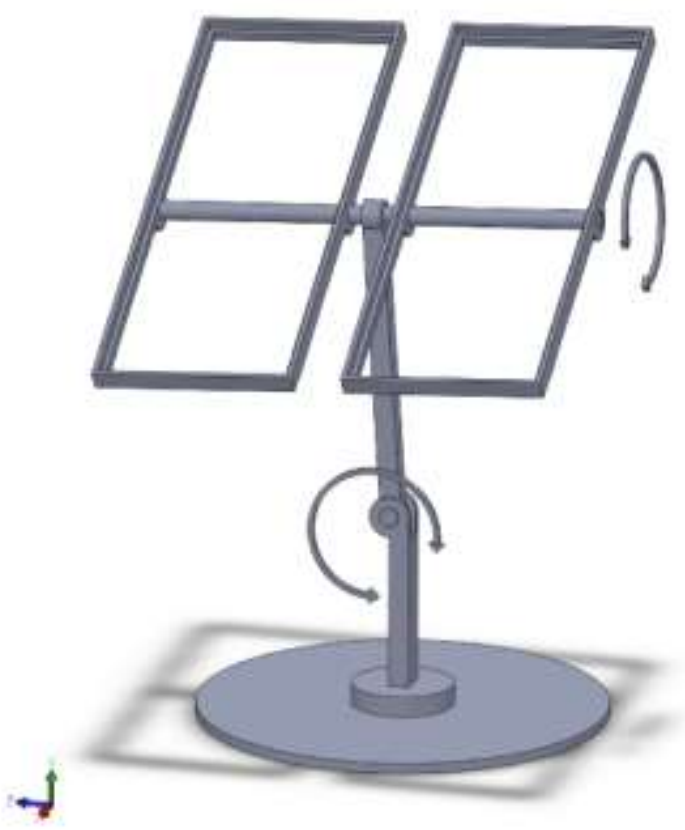

Fig. 1: Bi-axial tracking systems (a) azimuth (b) equatorial 
Table 1: Samples of angle values (elevation and azimuth)

$15 / 05 / 2017$

\begin{tabular}{lrr} 
Data hour & Elevation & Azimuth \\
\hline $04: 41: 25$ & $-0.812^{\circ}$ & $67.05^{\circ}$ \\
$05: 00: 00$ & $2.73^{\circ}$ & $74^{\circ}$ \\
$06: 00: 00$ & $14.98^{\circ}$ & $76.82^{\circ}$ \\
$07: 00: 00$ & $27.50^{\circ}$ & $83.95^{\circ}$ \\
$08: 00: 00$ & $40.13^{\circ}$ & $91.60^{\circ}$ \\
$09: 00: 00$ & $52.81^{\circ}$ & $101.02^{\circ}$ \\
$10: 00: 00$ & $64.98^{\circ}$ & $115.61^{\circ}$ \\
$11: 00: 00$ & $74.77^{\circ}$ & $146.66^{\circ}$ \\
$12: 00: 00$ & $75.65^{\circ}$ & $204.91^{\circ}$ \\
$13: 00: 00$ & $66.85^{\circ}$ & $240.98^{\circ}$ \\
$14: 00: 00$ & $54.98^{\circ}$ & $257.21^{\circ}$ \\
$15: 00: 00$ & $42.40^{\circ}$ & $267.17^{\circ}$ \\
$16: 00: 00$ & $29.71^{\circ}$ & $274.98^{\circ}$ \\
$17: 00: 00$ & $17.15^{\circ}$ & $282.14^{\circ}$ \\
$18: 00: 00$ & $4.90^{\circ}$ & $289.44^{\circ}$ \\
$18: 29: 03$ & $-0.812^{\circ}$ & $293.19^{\circ}$ \\
\hline
\end{tabular}

\section{Choice Criterion}

The need for stability and accuracy during orientation favours the azimuth tracker as the choice criterion of optimization, but the simultaneous solicitation of the correction of both angles (elevation and azimuth) makes it an energy consumer instead of an energy saver. To remedy this drawback, an almost perfect correction corrects the angular deviation due to the movement of the sun along the two angles (elevation and azimuth) as indicated in Fig. 2.

The choice of support for the energy optimization of a PV (Photovoltaic Panel) is an electromechanical system with azimuth orientation hybrid on its two spatial angles. These are calculated from an algorithm that takes into consideration both solar angles (elevation and azimuth). The operating principle of this hybrid technique is to combine two techniques (pre-programmed techniques and sensory techniques) profiting from their advantages in order to increase the efficiency of the PV by different phases of calculations, programming and dimensioning by minimizing the energy consumption during the movement.

In practical terms two tasks are performed; the first is to program the end positions of the tracker that corresponds to the sunrise and sunset as well as the correction angles and communicates with the different sensors using the same language in order to achieve a flexible control. The second is to make the control device autonomous if it is separated from the computer: This is why the microcontroller of the Arduino board is considered as a master of this tracking system but preferably the development of a Win32 (Windows 32-Bit Platform) application under the environment LabVIEW remains necessary for display reasons Fig. 3. The shape of the curve in the figure shows us the values of the solar declination during one year (2018), as well as the different values of the variables used to calculate the correction angles and the limit switches (sunrise and sunset).

This application presents the graphical interface between the computer and Arduino. Its task is to confirm the calculation of the correction angles of the panel from solar angles and to trace the different values collected by the sensors. It is therefore considered as a slave task.

The main algorithm implemented as a hexadecimal code on the Arduino using the LIFA extension takes the task of organizing the tracking control and favours the technique that should be selected during the correction starting with the pre-programmed technique at the beginning of the initialization.

At each degree of correction of the two angles (elevation and azimuth) the application receives a frame which carries three pieces of successive information: The motor to be activated, the direction of rotation and the duration equivalent to the angle of correction due to the deviation of the sun so as to be synchronized with the Arduino board. This connection is made through the USB port (Universal Serial Bus).

In addition, a measurement request is sent by the card to the sensors in order to acquire in real time the values of these quantities (voltage, current, irradiation and temperature). After processing the information received, the card selects: The number of the motor to be controlled, its direction of rotation, its delay for a double correction if there are errors. 


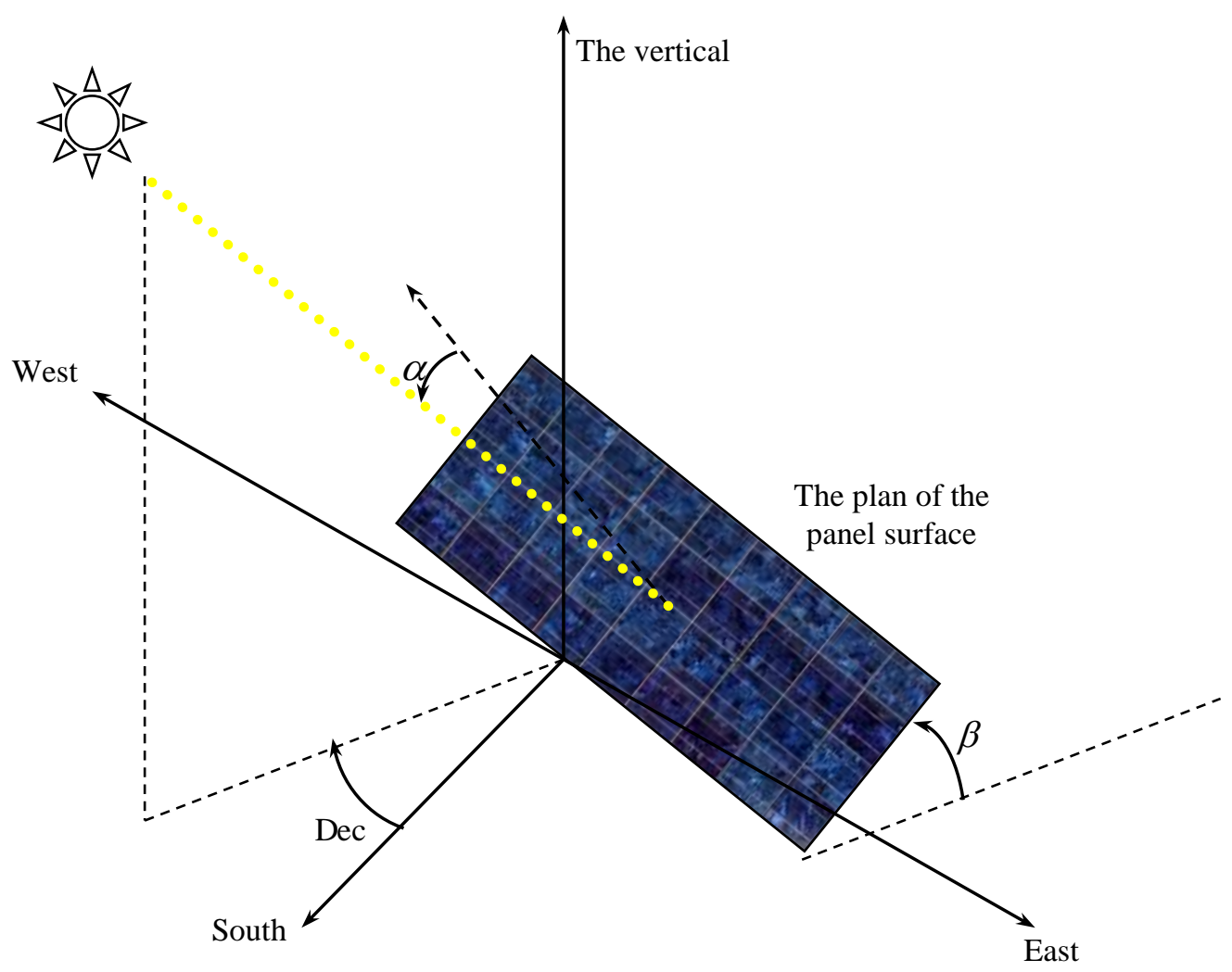

Fig. 2: The plan of the tracking axes

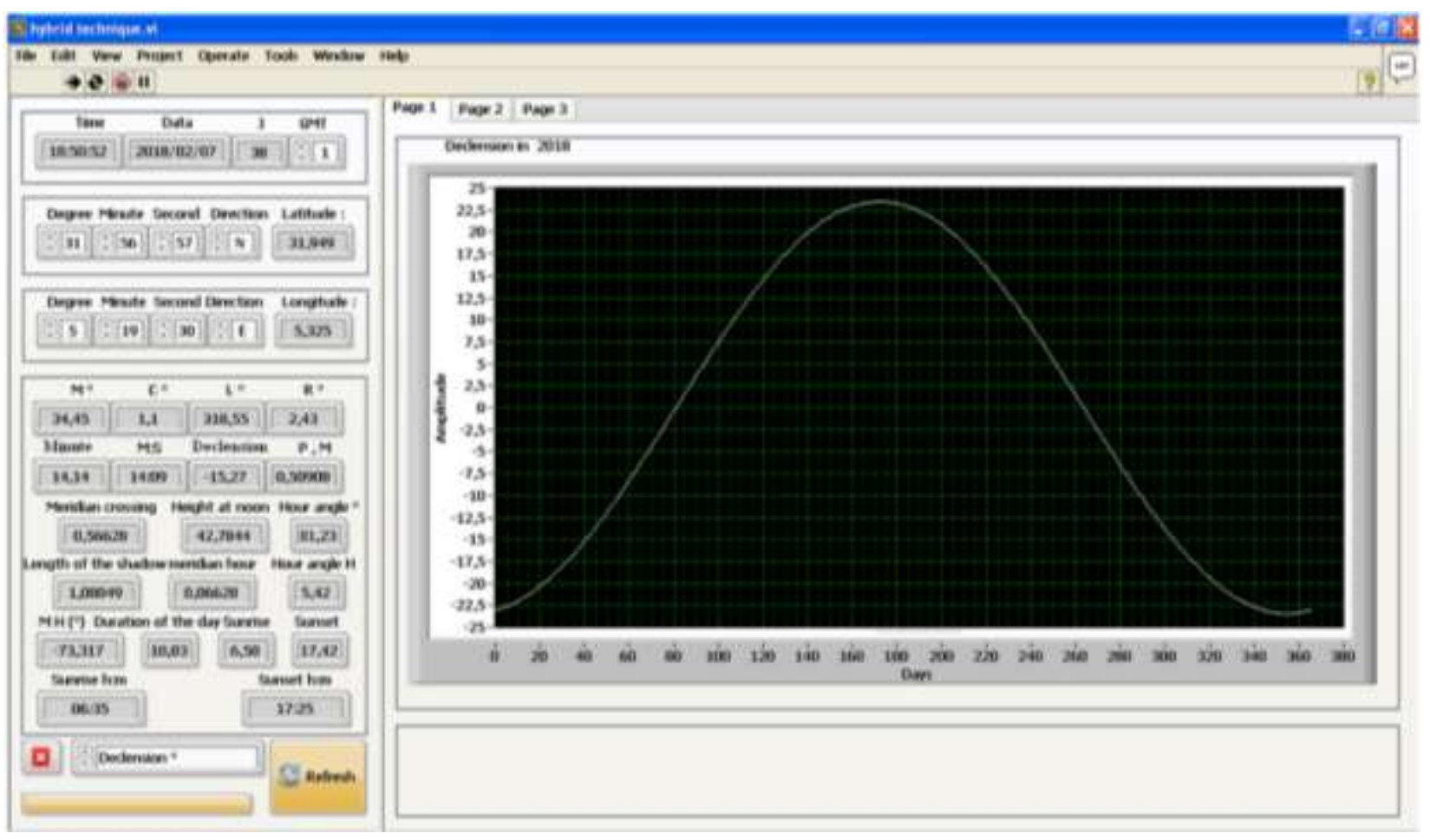

Fig. 3: The graphical interface of the hybrid technique under LabVIEW 


\section{General Structure of the Orientation Device Design}

The tracking system controls the movement of a photovoltaic module using a program in collaboration with sensors. The module is oriented using two axes to automatically follow the sun on a path defined by the equation of time translated into the program on Arduino. The Fig. 4 shows the overall system produced.

\section{Electrical Part}

The electrical board produced is essentially based on its control on an Arduino board whose role is to control the mechanical support of the panel via two DC (Direct Current) motors, the set of inputs and outputs as well as the calculation algorithm at the Arduino board presents the electrical tracking system. By using the algorithm that links between the sensors and the calculation results of the angles that become a database in the Electrically Erasable Programmable ReadOnly Memory (EEPROM) of the microcontroller, it is possible to achieve the hybrid technique. In order to benefit from the advantage of the sensory technique, four LDRs $\left(\mathrm{LDR}_{\mathrm{L}}, \mathrm{LDR}_{\mathrm{R}}, \mathrm{LDR}_{\mathrm{U}}, \mathrm{LDR}_{\mathrm{D}}\right)$ are used as inputs for the Arduino on four analog pins which are respectively $\left(A_{0}, A_{1}, A_{2}, A_{3}\right)$ in order to measure the intensity of the light in real time. These are positioned in the middle of each side of the panel support frame. Each one has a wall that separates it from the frame; the movements of the sun create an imbalance of the irradiation on the analog values of these LDRs which will trigger an interruption like second correction.

To determine and confirm the start and stop of the system as well as the cycle reset, two pushbuttons were positioned on the upper and lower ends of the panel frame. They are used as interruption pins on digital pins 4 and 5 .

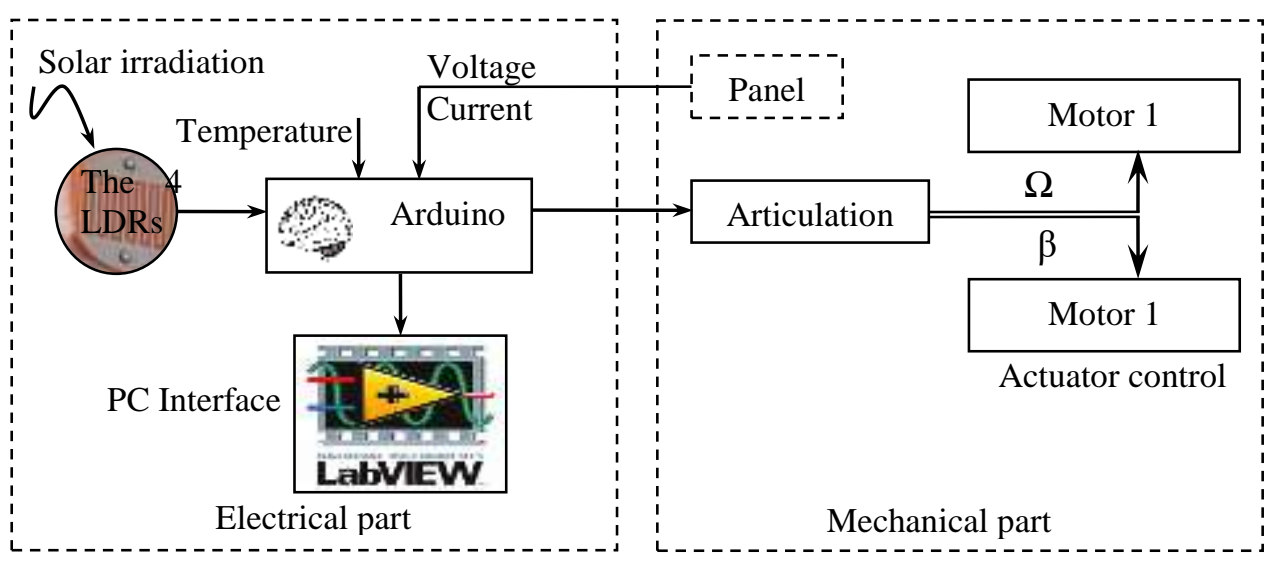

Fig. 4: The principle of the global tracking

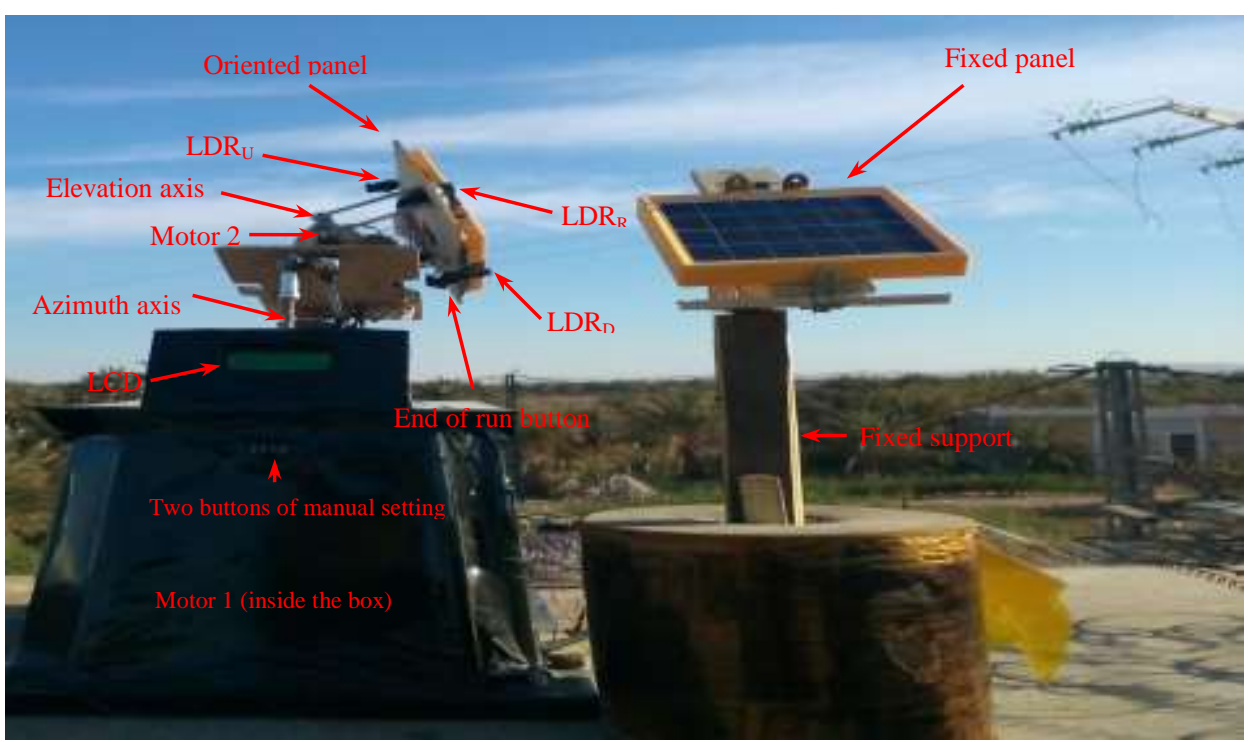

Fig. 5: The experimentation of the dual-axis hybrid device and fixed system 
The connection of the Arduino with an Liquid Crystal Display (LCD) $16 \times 2$ allows the display of any variation or internal and external action and makes the device independent and independent of the computer.

To follow the variation of the tension at pin $\mathrm{A}_{5}$, a simple assembly of two resistors is sufficient. To measure the intensity of the current generated by the solar panel, the ACS712 sensor was used on the $\mathrm{A}_{4}$ analog pin. Another LM35 sensor has been linked to the A 6 pin of the Arduino board in order to measure the value of the temperature.

The practical realization is illustrateda in Fig. 5 .

\section{Mechanical Part}

From the two solar angles (elevation and azimuth), the photovoltaic panel must be oriented on its own two angles (inclination $\beta$ and azimuth $\Omega$ ). For this, we mounted a mobile support for the panel equipped with two motor brains. The first motor is used to perform the correction of the azimuth angle $\Omega$. For the second motor brain, a system for converting circular motion into ranslational motion is mounted to provide the displacement $\mathrm{Z}$ equivalent to an inclination angle $\beta$. Figure 6 illustrates the mechanical part of the system produced.

The purpose behind adding the gears to the second motor brain is to slow down the movement or better control the correction of the angles with high precision. We choose two motor brains that can handle a weight of $4 \mathrm{Kg}$ since only test panels that do not weigh much are used.

The Table 2 shows the characteristics of the PV module used. It is considered as a mini prototype to perform hybrid tracking.

A drive mechanism for any tracking system often has an additional energy loss that must be drawn from the output power of the solar panel itself. The aim of the hybrid algorithm control strategy is to minimize the power consumed by the mechanism (articulation, motors) with an impulse command in order to improve the value of the net energy gain.

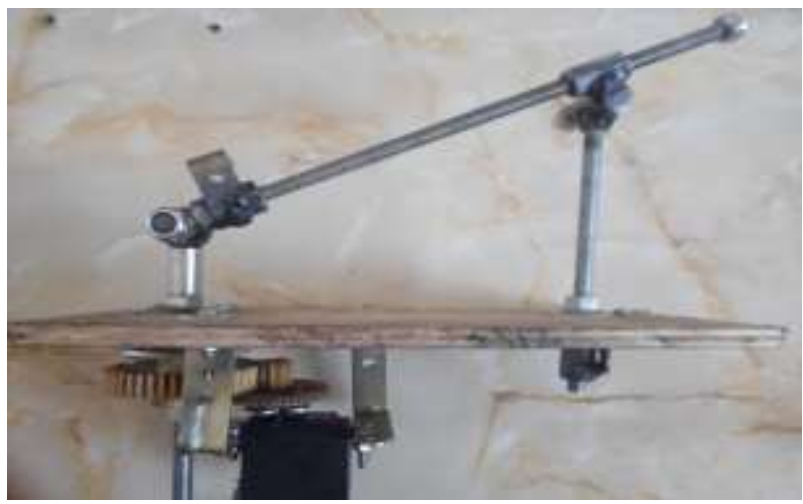

\section{Logarithmic Steps}

The development of the Hybrid Tracking Control algorithm is done under the LabVIEW environment of the company National Instruments (NI) using the LIFA extension which has the task of providing a development environment whose role is to simply allow the user to create virtual instruments using NI acquisition hardware to reproduce on a computer the behaviour of an instrument that uses: (A light intensity sensor, temperature sensor, a voltage sensor and a current sensor). The method is mainly dependent on the machine language that is suitable for handling a hexadecimal code closer to the Arduino microcontroller's core. The Fig. 7 shows the main block of the command through the LIFA extension.

The tracking control algorithm begins with the declaration of all variables used; after that, the ports of the Arduino are initialized as outputs, then; the timer sub-program already located on the microcontroller's core. The process can be executed through several stages.

\section{A- The Processing of the Elevation and Azimuth Angles of the PV}

This section depends mainly on the equations of time that determine the angles Declination and PV as well as the angle of the sunrise, after correction, the Arduino board enters sleep mode and it does not return to this equation until the next cycle (Sunrise).

Table 2: Characteristics of the used panel

\begin{tabular}{ll}
\hline Characteristics & values \\
\hline Weight & $309 \mathrm{~g}$ \\
Monocrystalline cells & $19 \%$ efficient \\
Open Circuit Voltage & $7.0 \mathrm{~V}$ \\
Peak Voltage & $6.0 \mathrm{~V}$ \\
Peak Current & $1500 \mathrm{~mA}$ \\
Peak Power & $9.0 \mathrm{Watt}$ \\
brand name & SR SOLAR \\
\hline
\end{tabular}

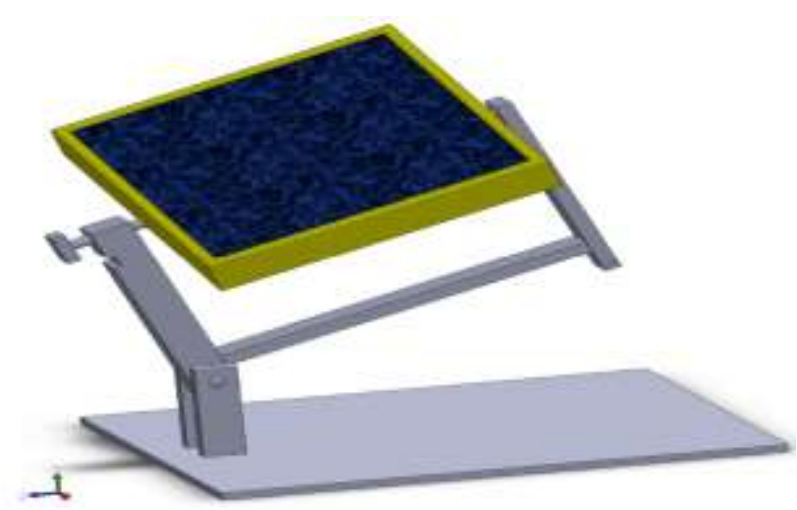

Fig. 6: The realized mechanical system 


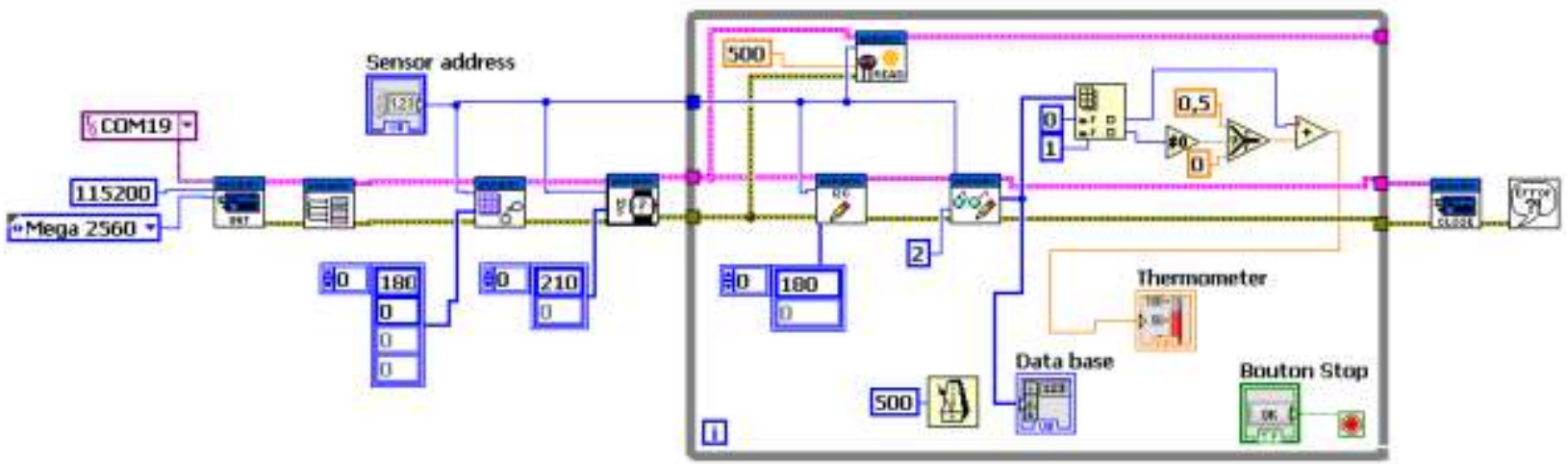

Fig. 7: The diagram of the hybrid Control around the Arduino

\section{B- Escapes for the Sunrise}

The purpose of this part is to set the VP at the starting point so that it moves to other parts only after verifying the conditions (Sunrise, The intensity of light is more than or equal to $500 \mathrm{Lux}$ ).

\section{C-Improvement of the Correction Angles of the PV}

This part is based on the light sensors that are the LDRs where the intensity of the light for each of the right Light Detect Resistor (LDR) and left LDR is measured; then the correction is done towards the trend of LDRs.

\section{D- Sun Tracking Operation}

This step depends on the LDR where the intensity of the light for each of the upper LDR and low LDR is measured. Re-measure the process again after three and a half minutes and put the Arduino in sleep mode during this time before re-measuring again.

\section{E- Functioning on the Confirmation of Sunset and Renewal of Beginning of PV Start}

To complete the $180^{\circ}$, an interruption occurs when the frame of the panel support touches a push button to declare the end of the race (sunset); the panel remains in the end point until the value of the intensity of light becomes less than 25 Lux then it returns to the starting point by a continuous movement in the opposite direction of the clock. The starting point algorithm is known by a second interrupt that is triggered when the panel support touches another push button.

The sun's rays take eight minutes during each trip. We have exploited three and a half minutes; it is an arithmetic mean between the longest day and the shortest day of the year.

\section{F-Tailored Operation}

Each time the panel is moved, a measurement phase is triggered in order to take the values of the quantities (voltage, current intensity, temperature and light intensity).

\section{Experimentation}

To demonstrate the efficiency of this hybrid method and the influence of the daily variation and the azimuthal variation of the sun on the solar panel efficiency, we present a comparison of the results obtained on a fixed module by to a two-axis oriented module based on the hybrid technique by correcting the elevation and azimuth variation that is directly related to the day and night variation and the seasonal variation under the same conditions. Afterwards, another comparison was made between the results obtained by the hybrid technique and the two techniques (pre-programmed, sensory). These experiments were carried out in one day (15/05/2017) where the irradiation was relatively good, and that south of Algeria (Ouargla) defined by its altitude, N31.963 and its longitude, E 5.342. The temperature has been variable during the day. For a daily correction, we corrected the solar displacement error with successive pulses corresponding to $3^{\circ}$. The control occurs by the mean of a periodic signal passing from the low state to the high state every three minutes. The command information is extracted from the database that is computed by the Arduino and stored in the EEPROM of the microcontroller. After each correction of the daily deviation, a deviation error is added and disturbs the value of the angle of elevation (the variation of the path that the sun follows during the year). For this, we added a second correction that relates to this angle to intervene the LDRs sensors, which gives us the true position correcting the error if it is produced. In the day when the measurements were taken: Voltage measurements and current intensity are made at equal time intervals. The Copernicus equation gives us an initial declination of the photovoltaic module at $-16.40^{\circ}$. The graphical interface of the LabVIEW environment allowed the display of the experimental curves of the quantities: Current and voltage on the two situations: Fixed module and two-axis system. The results are shown in the Fig. 8-10. 


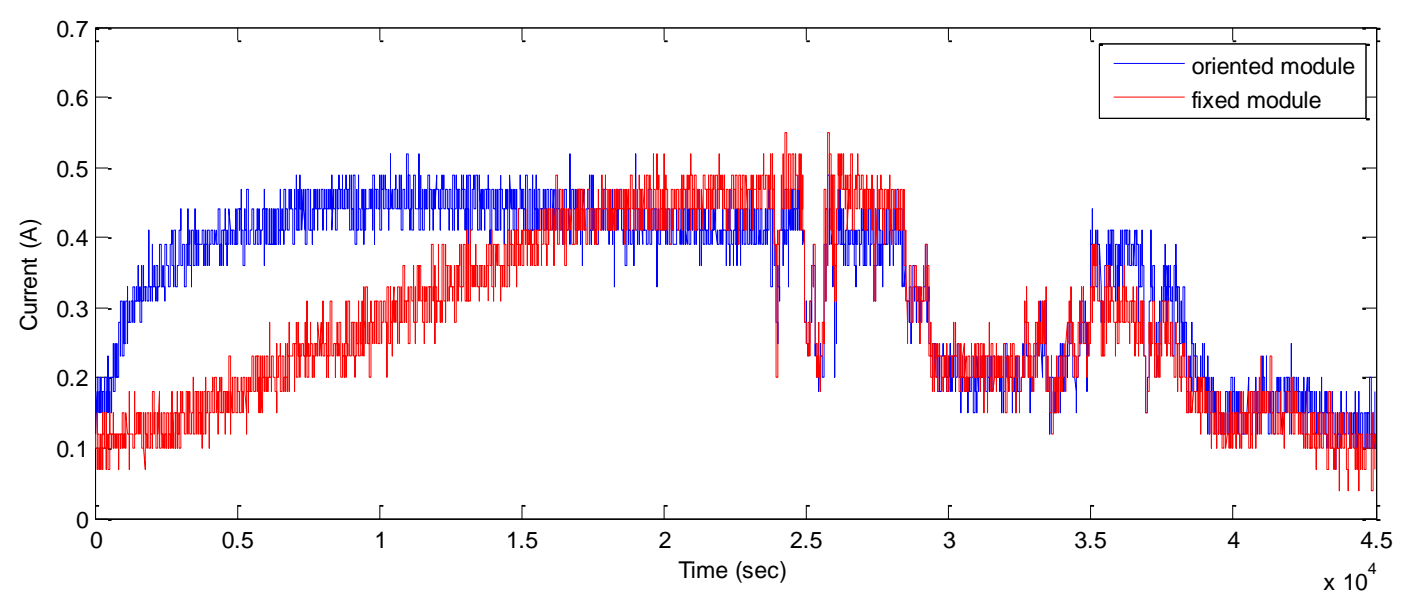

Fig. 8: Current-time curves for a fixed module and an oriented module
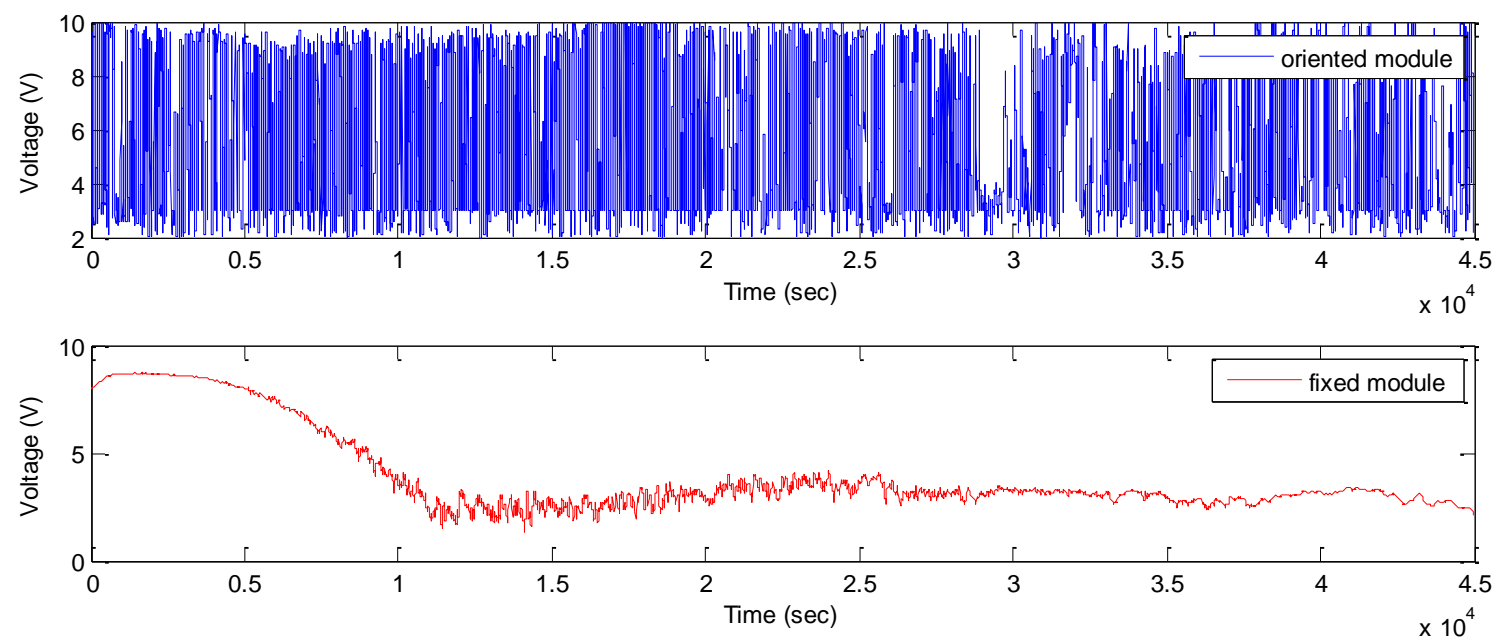

Fig. 9: Voltage-time curves for a fixed module and an oriented module
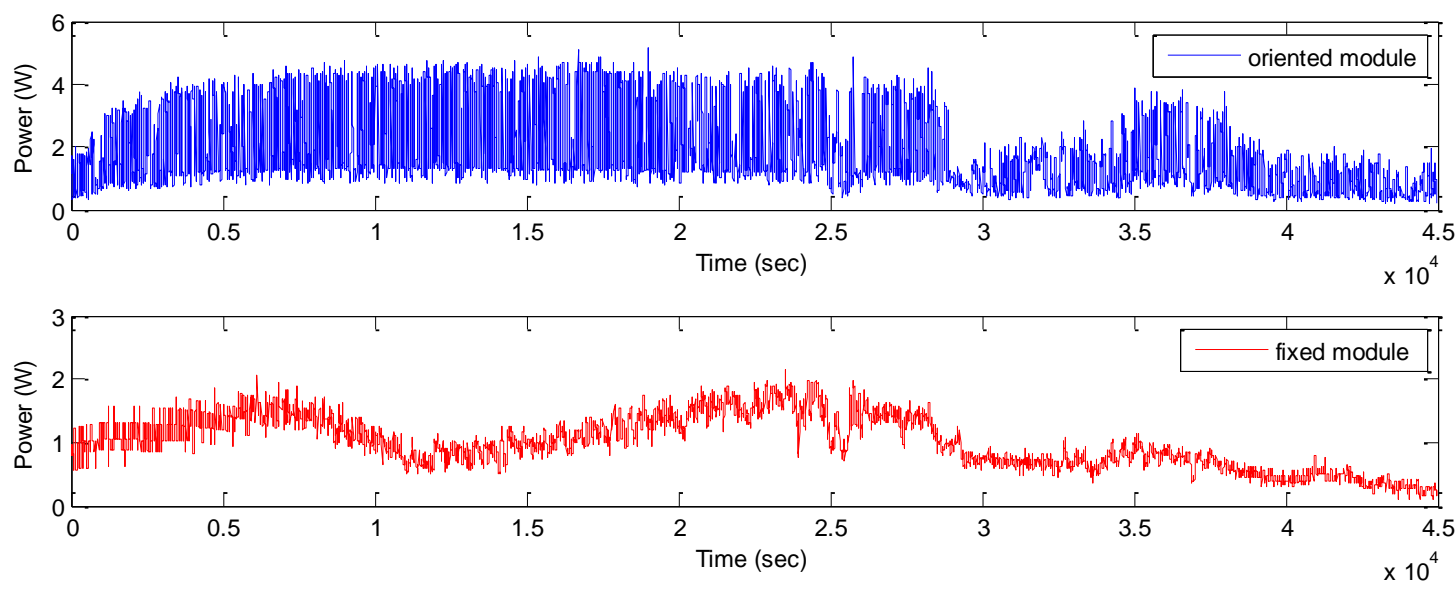

Fig. 10: Power-time curves for a fixed module and an oriented module 
The surface difference between the two curves for the power curve is about $36 \%$. It represents the gain obtained by the correction and shows the importance of this parameter on the efficiency of a photovoltaic module. The direct relationship between the luminous flux and the output current intensity of the photovoltaic panel summarizes that the luminous flux stability implies the stability of the current value and the increase or decrease of the luminous flux causes the increase or decrease of current as shown in Fig. 11.

To prove the efficiency of the hybrid tracking over both pre-programmed and sensory techniques, we have experimentally deducted the values of the quantities I and $\mathrm{V}$ in order to plot the power delivered by the panel by deactivating the hybrid algorithm during the correction and select one of the two techniques as well as their commands to be able to draw the power curve and that on the same graph to compare them and extract the energy gained by the best technique.
In order to validate the performances of the hybrid technique by comparing the two pre-programmed and sensory techniques, the measurements of the quantities I and $\mathrm{V}$ were carried out and the power was plotted on two charts: The first is the curves of the power harvested by the hybrid technique and the pre-programmed technique. The second is the curves of the power of the hybrid technique with sensory technique. To compare the power plotted by LabVIEW on the same chart and at the same operating conditions on the same day, the angular deviation is corrected by the hybrid technique and the values of the $\mathrm{I}$ and $\mathrm{V}$ quantities are linked to plot the power. Then, the panel is repositioned and this technique is deactivated and then the difference is corrected another time by one of the two techniques to take again the values of $\mathrm{I}$ and $\mathrm{V}$ to plot the power delivered by this technique and the cycle is repeated again. The Fig. 12 anf Fig. 13 present the comparison of the powers of the hybrid technique with the two other techniques.

The surface difference between the two plots for the power curve is about $2 \%$.
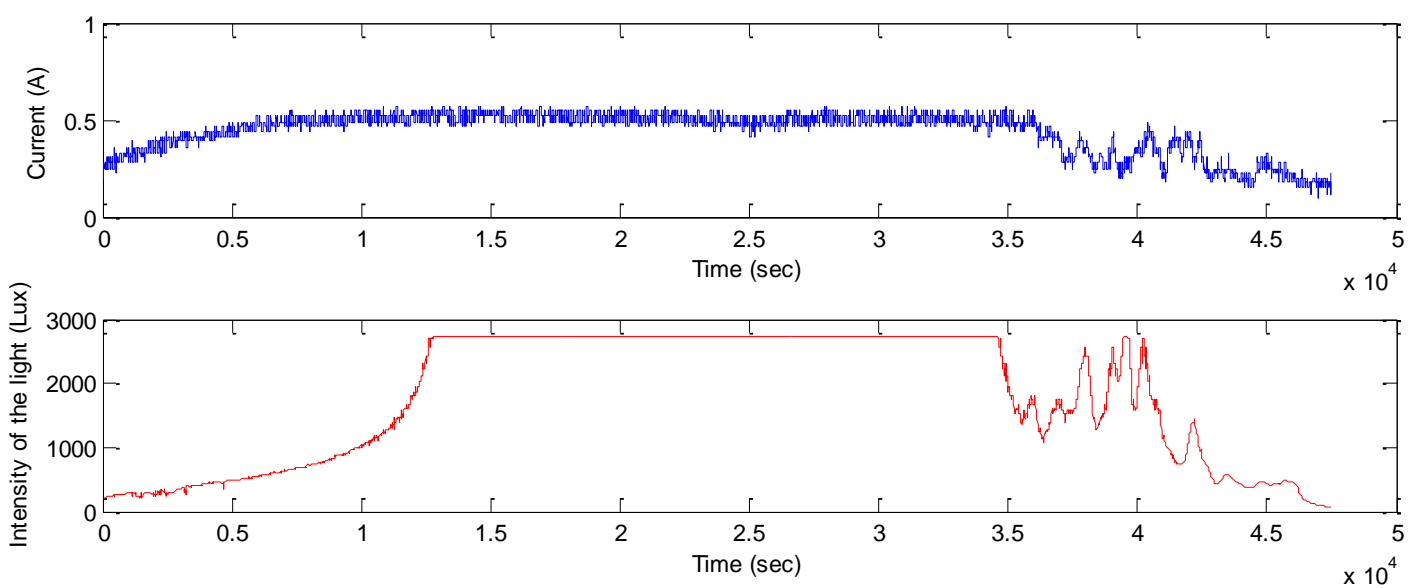

Fig. 11: The relationship between the output current of the oriented panel and the intensity of the light

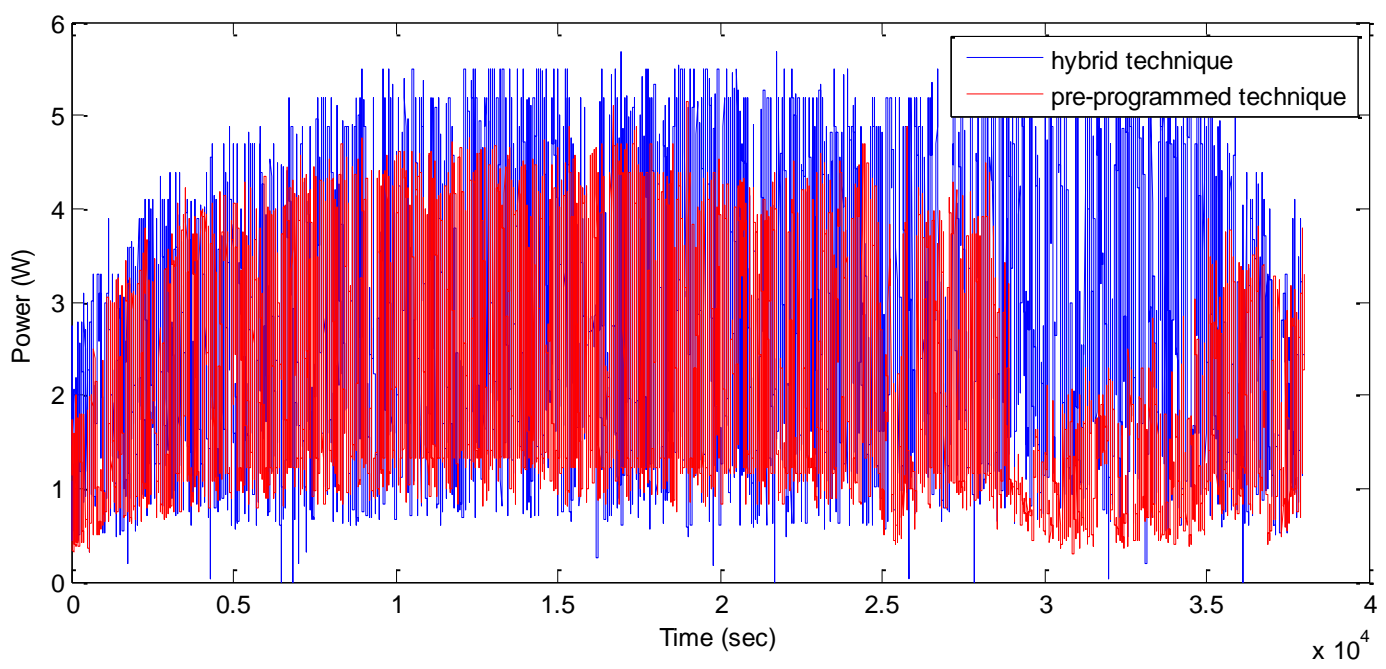

Fig. 12: Power-time curves of the hybrid technique and the pre-programmed technique (19/06/2017) 


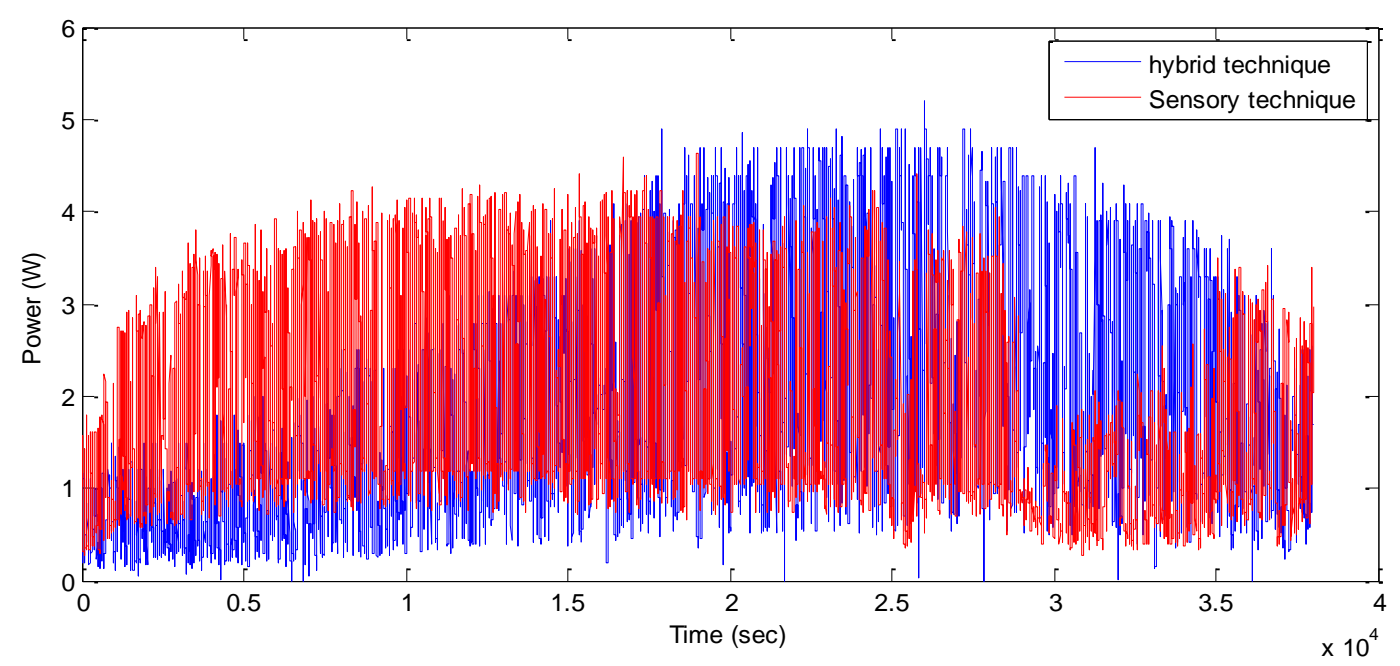

Fig. 13: Time-power curves of the hybrid technique and the sensory technique (20/06/2017)

The surface difference between the two plots for the power curve is about $2.3 \%$.

\section{Conclusion}

The work proposes a new approach concerning the optimization of photovoltaic systems obtained by designing and implementing a two-axis hybrid tracking system for a photovoltaic panel. The tracking is provided by a hybrid technique that transmits pulses to two actuators that orient the panel in a way the angle of incidence remains constant.

Pulse rate characterises the way of the transmission of the correction towards the actuators in order to optimize the duration of the movement.

Comparing the results obtained by the hybrid technique with that of the two pre-programmed and sensorial techniques. The angular correction is done for an optimal time and the additional energy gain collected is of the order of 2 and $2.3 \%$ respectively.

This technique is a mixture of two major techniques known as pre-programmed and sensory technique. Its purpose is to gain profit from their advantages and eliminate their disadvantages during the correction period. The double correction, the precision and the stability of pursuit as well as the exploitation of the optimal duration of sunshine are the major goals of this technique. The use of virtual simulation as a start and the collaboration with the LabVIEW environment facilitated the task of practical realization. The use of an embedded mini system that is the Arduino board has made the device autonomous.

This technique makes it possible to exploit solar energy in an efficient way not only during the hours of sunshine but also the light diffused throughout the year. This system has improved energy efficiency by $36 \%$. The experimental results confirm that the benefit of two-axis tracking is very advantageous and is incommensurate compared to the insignificance of the investments involved.

\section{Acknowledgement}

Works have been supported by the authors themmselves.

\section{Author's Contributions}

Mohamed Redha Rezoug: Conceived and designed the experiments and performed them; analyzed the data; contributed reagents, materials and analysis tools wrote the paper.

Rachid Chenni: Conceived and designed the experiments, performed them, analyzed the data and participated in writing the paper.

\section{Ethics}

The authors confirm that there are no ethical issues involved in this article and that the latter contains original unpublished material.

\section{References}

Alexandru, C. and I.N. Tatu, 2013. Optimal design of the solar tracker used for a photovoltaic string. J. Renewable Sustainable Energy, 5: 1-16. DOI: $10.1063 / 1.4801452$

Arslan, A.R., K. Addoweesh, A. El-Leathy and H. Al-Ansary, 2014. Sun position algorithm for sun tracking applications. Proceedings of the 40th Annual Conference of the Industrial Electronics Society, Oct. 29-Nov. 1, IEEE Xplore Press, Dallas, TX, USA. DOI: 10.1109/IECON.2014.7049356 
Carlos, M., F. Daniel, S. Pablo, V. Gabriela and P.D. Jorge, 2017. New prototype of photovoltaic solar tracker based on arduino. Energies, 10: 1-13.

DOI: $10.3390 /$ en 10091298

Cheng, T C., W.C. Hung, and T.H. Fang, 2013. Twoaxis solar heat collection tracker system for solar thermal applications. Int. J. Photoenergy, 2013: 1-7. DOI: 10.1155/2013/803457

Chong, K.K. and C.W. Wong, 2009. General formula for on axis sun tracking system and its application in improving tracking accuracy of a solar collector. Solar Energy, 83: 298-305.

DOI: 10.1016/j.solener.2008.08.003

Eke, R. and A. Senturk, 2012. Performance comparison of a double axis sun tracking versus fixed PV system. Solar Energy, 86: 2665-2672.

DOI: 10.1016/j.solener.2012.06.006

Fabienne, S., G. Alberto, T. José-Luis. and P. Ramón, 2015. Optical losses due to tracking error estimation for a low concentrating solar collector. Energy Conversion Manage., 92: 194-206.

DOI: 10.1016/j.enconman.2014.12.026

Radu, V. and L. Mihai, 2014. Torques on rotational axes of PV azimuthal sun tracking systems. Springer Proc. Energy, 10: 461-470.

Saban, Y., R. Hasan, D. Osman, D. Furkan and A. Oguzhan et al., 2015. Design of two axes sun tracking controller with analytically solar radiation calculations. Renewable Sustainable Energy Rev., 43: 997-1005. DOI: 10.1016/j.rser.2014.11.090

Salsabila, A., S. Suhaidi and A.A.K Mohd-Zainal, 2013. Power feasibility of a low power consumption solar tracker. Proc. Environ. Sci., 17: 494-502.

DOI: 10.1016/j.proenv.2013.02.064
Senpinar, A. and M. Cebeci, 2012. Evaluation of power output for fixed and two-axis tracking PV arrays. Applied Energy, 92: 677-685. DOI: 10.1016/j.apenergy.2011.07.043

Walsh, A., D. Cóstola and L. Chebel, 2017. Review of methods for climatic zoning for building energy efficiency programs. Build. Environ., 112: 337-350. DOI: 10.1016/j.buildenv.2016.11.046

Ya'u, M.J., 2017. A Review on solar tracking systems and their classifications. J. Energy, Environ. Chem. Eng., 2: 46-50. DOI: 10.11648/j.jeece.20170203.12

Yougeshwarsingh, R. and O. Vishwamitra, 2014. Improving the dual axis solar tracking system efficiency via drive power consumption optimization. Applied Solar Energy, 50: 74-80. DOI: $10.3103 / \mathrm{S} 0003701 \mathrm{X} 1402011 \mathrm{X}$

Zhang, P., G. Zhou, Z. Zhu, W. Li and Z. Cai, 2013. Numerical study on the properties of an active sun tracker for solar streetlight. Mechatronics, 23: 1215-1222. DOI: 10.1016/j.mechatronics.2013.08.007

$\begin{array}{ll}\text { Abbreviations } \\ \text { LIFA: } & \text { LabVIEW interface for Arduino } \\ \text { PV: } & \text { Photovoltaic } \\ \text { USB: } & \text { Universal Serial Bus } \\ \text { LDR: } & \text { Light Detect Resistor } \\ \text { LabVIEW: } & \text { Laboratory Virtual Instrument Engineering } \\ & \text { Workbench } \\ \text { LCD: } & \text { Liquid Crystal Display } \\ \text { EEPROM: } & \text { Electrically Erasable Programmable Read- } \\ & \text { Only Memory } \\ \text { Win32: } & \text { Windows 32-Bit Platform } \\ \text { DC: } & \text { Direct Current } \\ \text { NI : } & \text { National Instruments }\end{array}$

